Bundesgesundheitsbl 2012 $\cdot 55: 1057-1060$ DOI 10.1007/s00103-012-1508-1

(c) Springer-Verlag 2012
Mitteilungen des Arbeitskreises Blut des Bundesministeriums für Gesundheit

\section{XMRV ist nicht human- pathogen und hat keine Bedeutung für die Sicherheit von Blut und Blutprodukten}

\author{
Stellungnahmen des Arbeitskreises Blut \\ des Bundesministeriums für Gesundheit
}

Der Arbeitskreis Blut des Bundesministeriums für Gesundheit gibt als nationales Beratungsgremium Stellungnahmen zu neuartigen Erregern ab, bewertet neue Erkenntnisse zu bekannten Erregern und erarbeitet entsprechende Empfehlungen für die Fachöffentlichkeit. Diese Serie von Stellungnahmen zu einzelnen Erregern werden als Zusammenfassung des aktuellen Wissensstandes veröffentlicht, speziell unter transfusionsmedizinisch relevanten Aspekten (Bundesgesundhbl., 41, 53, 1998).

Frühere Beiträge befassten sich mit der Creutzfeldt-Jakob-Erkrankung, dem Parvovirus B19 und dem GB-Virus Typ $C$ (Hepatitis-G-Virus) (Bundesgesundheitsbl., 41, 78-90, 1998), HTLV-I/-II (Bundesgesundheitsbl., 41, 512, 1998), Yersinia enterocolitica (Bundesgesundheitsbl., 42, 613, 1999), TT-Virus (Bundesgesundheitsbl., 43, 154-156, 2000), Hepatitis- $B$-Virus (HBV) (Bundesgesundheitsbl., 43, 240-248, 2000), Humanes Cytomegalovirus (HCMV), (Bundesgesundheitsbl., 43, 653-659, 2000), Hepatitis-A-Virus (Bundesgesundheitsbl., 44, 844-850, 2001), Treponema pallidum (Bundesgesundheitsbl. 45, 818-826, 2002), Hepatitis-C-Virus (Bundesgesundheitsbl. 46, 712-722, 2003), Humanes Immunschwächevirus (HIV) (Bundesgesundheitsbl. 47, 83-95, 2004), Arboviren - durch Arthropoden übertragba- re Viren (Bundesgesundheitsbl. 47, 910918, 2004), Coxiella burnetii - Erreger des Q-(query) Fiebers (Bundesgesundheitsbl. 48, 814-821, 2005), Variante Creutzfeldt-Jakob-Krankheit (Bundesgesundheitsbl. 48, 1082-1090, 2005), Influenzaviren (Bundesgesundheitsbl. 50, 11841191, 2007), Arbobakterien (über Arthropoden übertragbare Bakterien) (Bundesgesundheitsbl. 50, 1192-1207, 2007), Hepatitis-E-Virus (Bundesgesundheitsbl. 51, 90-97, 2008), Malaria (Bundesgesundheitsbl. 51, 236-249, 2008), Arboprotozoen (Bundesgesundheitsbl. 52, 123-146, 2009), Orthopockenviren: Infektionen des Menschen (Bundesgesundheitsbl. 53, 957-972, 2010), Humanes Cytomegalievirus (HCMV) (Bundesgesundheitsbl. 53, 973-983, 2010), Parvovirus B19 (Bundesgesundheitsbl. 53, 944-956, 2010), Dengue Fieber Virus (DENV) (Bundesgesundheitsbl. 54, 892-903, 2011), Arbonematoden - durch Arthropoden übertragbare Nematoden-Infektionen (Bundesgesundheitsbl. 55, 1044-1056) und WestNil-Virus (Bundesgesundheitsbl. 55, 1024-1043).

\section{Das neu beschriebene Retro- virus XMRV ist kein human- pathogenes Virus und hat keine Bedeutung für die Sicherheit von Blut und Blutprodukten}

\section{Zusammenfassung}

Im Jahr 2009 wurde berichtet, dass ein neues Virus, das Retrovirus XMRV, Ursache für das chronische Müdigkeitssyndrom (CSF) und für Prostata-Krebs sei. Es entstand der Verdacht, dass XMRV, wie auch andere Retroviren, durch Bluttransfusionen übertragbar sein könnte. Weitere eingehende Untersuchungen ergaben jedoch, dass die anfänglich erhobenen Befunde nicht aufrechterhalten werden konnten. Sie waren auf Verunreinigungen im Labor zurückzuführen. Nach derzeitigem Erkenntnisstand ist XMRV kein humanes Retrovirus und ist nicht auf den Menschen übertragbar.

\section{Ursprüngliche Einschätzung von XMRV als neues Retrovirus mit humanpathogenem Potenzial}

Humane Retroviren können durch Blut und Blutprodukte übertragen werden. Das Risiko der Übertragung der Immunschwächeviren HIV-1 und HIV-2 ist durch Maßnahmen zur Spenderauswahl und -testung für nicht inaktivier- 
te Blutkomponenten in Deutschland auf kleiner als 1:5.000.000 reduziert worden. Durch Verfahren zur Abtötung oder Entfernung von Viren werden diese Infektionen durch Plasmaderivate nicht mehr übertragen.

Auf der Suche nach weiteren humanpathogenen Retroviren wurde im Jahr 2006 ein exogenes humanes Retrovirus in klinischem Probenmaterial beschrieben [1]. Dieses als Xenotropic Murine Leukemia Virus-related Virus (XMRV) bezeichnete Retrovirus wurde mit Hilfe eines Mikroarrayverfahrens in Prostatakarzinombiopsien US-amerikanischer $\mathrm{Pa}$ tienten nachgewiesen. In nachfolgenden Veröffentlichungen wurde XMRV in bis zu 27 \% der untersuchten Prostatakarzinome diagnostiziert [2].

Neben einer Assoziation mit Prostatakarzinomen, einer der häufigsten Tumorerkrankungen des Mannes, wurde XMRV in einer weiteren Arbeit aus den USA in mehr als 2/3 aller Patienten mit Chronischem Erschöpfungssyndrom (Chronic Fatigue Syndrome, CFS) beschrieben [3]. Schätzungen gehen davon aus, dass in Deutschland etwa 300.000 Personen an diesem Syndrom leiden. Da die Ursache dieses Krankheitsbildes bisher unbekannt ist, existiert auch keine kausale Therapie. Die Diagnose erfolgt letztlich durch Ausschluss anderer Erkrankungen mit ähnlicher Symptomatik.

Die Autoren der Studie über CSF-Patienten um Judy Mikovits vom Whittemore Peterson Institute (WPI) in Reno, USA, und dem National Cancer Institute (NCI) in Frederick, USA, berichten in ihrer Veröffentlichung von erfolg reichen Isolierungen des Virus aus dem Blut positiv getesteter CFS-Patienten, der Infektion frischer Blutzellen gesunder Spender und dem Nachweis spezifischer Antikörper. Die Spezifität der Antikörper wird jedoch nicht mit einem Westernblot bestätigt, wie er z. B. in der HIV- oder HTLV-Diagnostik üblich ist. Als bemerkenswert wurde außerdem die mit 3,7 \% sehr hohe Prävalenz des Virus im Blut gesunder Kontrollen angesehen.

In einer weiteren Arbeit haben Wissenschaftler des National Institute of Health (NIH) und der Food and Drug Administration (FDA) Sequenzen XMRV-verwandter Viren (sogenannter polytro- per und modifiziert polytroper muriner Leukämieviren) im Blut von fast $90 \%$ der untersuchten CFS-Patienten und bei über $6 \%$ der gesunden Blutspender diagnostiziert [4]. XMRV wurde in dieser Studie jedoch nicht direkt nachgewiesen und es blieb offen, ob infektiöse Viren vorhanden waren.

Die in den Studien beschriebene signifikante Assoziation von XMRV mit dem Prostatakarzinom und CFS sind allein noch kein Beleg für eine kausale Rolle bei diesen Erkrankungen. Das Virus könnte ein harmloser Opportunist sein, dem pathophysiologische Umstände eine persistierende Infektion ermöglichen. Zu bedenken ist in diesem Zusammenhang jedoch, dass durch die Integration eines Retrovirusgenoms in das Genom einer Wirtszelle Tumore induziert werden können (Insertionsmutagenese). So ist z.B. bekannt, dass das Retrovirus HTLV Lymphome und Leukämien, aber auch neurologische Erkrankungen in einem Teil der Infizierten verursacht.

Ausgehend von diesen Erkenntnissen könnte XMRV ebenfalls sowohl an der Ätiologie maligner als auch neurologischer Erkrankungen beteiligt sein. Angesichts der Tatsache, dass vergleichbare Retroviren Tumore in Nagern verursachen und im Zuge gentherapeutischer Applikationen als Vektorsystem auch im Menschen zu Krebserkrankungen geführt haben, wurde zu dem Zeitpunkt von einem erheblichen kanzerogenen Potential des XMRV bei einer Infektion des Menschen ausgegangen. Des Weiteren wurde in Rhesusaffen gezeigt, dass XMRV in der Lage ist, eine chronisch persistierende Infektion zu etablieren [5].

\section{Neuere kontroverse Ergebnisse zur Prävalenz und Krankheits- assoziation von XMRV}

Nach der Publikation der oben genannten Arbeiten erschienen Untersuchungen anderer Arbeitsgruppen. Einzelne Arbeitsgruppen beschrieben ebenfalls XMRV sowohl in Prostatatumoren als auch in anderen klinischen Materialien, darunter waren auch Arbeiten aus Deutschland [6,7]. In der Mehrzahl der später veröffentlichten Studien wurde XMRV jedoch in keiner einzigen Probe nachgewiesen $[8,9$,
10, 11, 12, 13]. Bei Wiederholungsuntersuchungen von zuvor am WPI im Rahmen der initialen Studie [3] positiv getesteten CFS-Patienten konnten in unabhängigen Laboren in keinem einzigen Fall XMRV oder spezifische Antikörper gegen XMRV-Proteine nachgewiesen werden, obwohl nahezu identische, hochsensitive Verfahren verwendet wurden $[14,15]$. Hiermit stellt sich die Frage, was die Ursache für den beschriebenen Nachweis von XMRV am WPI und an einem kommerziellen Labor sowie in den anderen Studien sein könnte.

\section{Kontaminationen als Quelle positiver XMRV-Befunde}

Bereits in den Publikationen der ersten negativen Studienergebnisse und den Kommentaren dazu wurden Kontaminationen als mögliche Ursache der XMRVNachweise im Blut und anderem klinischen Material diskutiert. Da man davon ausging, dass nur geringe Mengen Virus in klinischen Proben vorhanden sind, wurden in der Regel ultrasensitive und damit besonders kontaminationsanfällige PCR-basierte Nachweisverfahren eingesetzt. Meist waren diese Methoden jedoch nicht in der Lage zwischen endogenen retroviralen Sequenzen im Mausgenom und XMRV zu unterscheiden. Mehrere Arbeitsgruppen, die positive XMRV-Befunde erhalten hatten, konnten zeigen, dass ihre positiven Ergebnisse auf Kontaminationen mit muriner genomischer DNA zurückzuführen sind $[16,17]$. Die Quelle der Verunreinigung konnte in einigen Fällen identifiziert werden [15]. In DNA-Extraktionssäulen und in kommerziellen Polymerasepräparationen wurden Spuren muriner DNA nachgewiesen, die möglicherweise durch monoklonale Antikörper bei Reinigung der Polymerase eingebracht wurden $[18,19]$.

Als weitere Kontaminationsquellen werden klonierte XMRV-DNA und mit XMRV infizierte Zelllinien angesehen. Dieser Verdacht konnte in einem Fall durch Analyse der Integrationsorte bestätigt werden [20]. Für die Annahme einer Kontamination spricht auch die hohe Identität der publizierten XMRV-Sequenzen, die aus den verschiedenen kli- 
nischen Materialien von Prostatakarzinom- und CFS-Patienten stammen [21]. Bei einem Gammaretrovirus (zu denen auch XMRV gruppiert wird), das in der menschlichen Population zirkuliert, wäre eine höhere Heterogenität zu erwarten gewesen. Phylogenetische Analysen lassen den Schluss zu, dass sämtliche XMRV-Sequenzen aus einer einzigen Quelle stammen - der Zelllinie 22Rv1 [21].

\section{Entstehung von XMRV in einer in Mäusen etablierten humanen Prostatakarzinom Zelllinie}

In einer kürzlich publizierten Arbeit wurde die Entstehung von XMRV aufgeklärt [22]. Das Virus ist im Zuge der Etablierung der Prostatakarzinomzelllinie 22Rv1 [23] in einer Labormaus in Cleveland, Ohio, entstanden. Bei diesen Arbeiten wurden Prostatatumorzellen in athymischen Nacktmäusen passagiert. Dabei ist durch eine Rekombination zweier endogener retroviraler Sequenzen der Maus das xenotrope Retrovirus XRMV entstanden [22]. XMRV ist nicht in der Lage, Zellen der Maus zu infizieren, da die Labormaus keinen passenden Zellrezeptor exprimiert. XMRV konnte jedoch in den Zellen des humanen Xenotransplantats replizieren, da humane Zellen den entsprechenden Zellrezeptor besitzen. Der Zeitpunkt der Entstehung von XMRV lässt sich in etwa auf das Jahr 1996 eingrenzen, denn nur spätere Passagen des humanen Tumors enthalten das Virus [22]. Da die beiden Vorläufersequenzen identifiziert wurden, kann das Rekombinationsereignis, das zu XMRV geführt hat, eindeutig nachvollzogen werden. Die Wahrscheinlichkeit, dass ein solches Rekombinationsereignis unabhängig davon ein zweites Mal stattgefunden hat, ist mit etwa $10^{-12}$ extrem gering [22]. Heute gilt als gesichert, dass alle XMRV-Sequenzen aus der 22Rv1-Zelllinie stammen. Unter Berücksichtigung der oben angeführten Argumente stellt sich daher die Frage nach der Validität der Untersuchungsergebnisse, die in den Arbeiten über positive XMRV-Diagnosen in Patientenproben beschrieben wurden [3].
XMRV: Kein Risiko für

die Sicherheit von Blut

und Blutprodukten

Die unterschiedlichen Untersuchungsergebnisse in den verschiedenen Studien haben zu einer kontroversen Diskussion geführt. Gegenwärtig sprechen folgende Erkenntnisse dagegen, dass XMRV ein humanes Retrovirus ist oder in der menschlichen Population zirkuliert:

- Es gibt keine unabhängige Bestätigung des XMRV-Nachweises im Blut von CFS-Patienten und gesunden Kontrollen.

- Unabhängige Nachuntersuchungen von zuvor von Mikovits und Mitarbeitern als XMRV-positiv deklarierten CFS-Patienten konnten die ursprünglichen Ergebnisse nicht bestätigen.

- Viele der neueren Studien können durchgängig kein XMRV in Prostatatumoren bzw. Blutproben von CFSPatienten nachweisen.

- Die Originalarbeit zur Assoziation von XMRV und CFS wurde daher später mit einem „Expression of Concern“ durch den Editor der Fachzeitschrift versehen [24].

- Kontaminationen von diagnostischen Reagenzien mit muriner DNA oder während der Probenaufarbeitung haben zu falsch-positiven Ergebnissen geführt.

- Die hohe Übereinstimmung aller bisher bekannten XMRV-Sequenzen legt den Ursprung des Virus aus nur einer weit verbreiteten Zelllinie (22Rv1) nahe.

Nach den ersten Veröffentlichungen zu XMRV im Jahr 2010 haben Kanada, Australien, Neuseeland, England sowie die USA CFS-Patienten von der Blutspende ausgeschlossen, um die wissenschaftliche Klärung der XMRV-Assoziation mit Erkrankungen des Menschen abzuwarten. Jedoch weder in diesen noch in anderen Staaten werden gegenwärtig Blut- oder Organspenden auf XMRV getestet.

Bis heute fehlen epidemiologische oder andere Hinweise auf eine Assoziation von Prostatatumorerkrankungen oder CFS mit Bluttransfusionen. Zudem ist kein einziger Fall einer Mensch-zu-Mensch-
Übertragung des Virus bei Transfusionen oder über einen anderen Infektionsweg bekannt.

Die Einführung von XMRV-Nachweisverfahren und das Screening von Blutspenden oder Transplantaten sind derzeit aufgrund der vorliegenden wissenschaftlichen Erkenntnisse nicht angezeigt. Der Arbeitskreis Blut sieht deshalb keine Veranlassung, die aktuellen Richtlinien, Empfehlungen und Spenderauswahlkriterien zu ändern.

Dieses Papier wurde fertig gestellt am 22.11.2011 und vom Arbeitskreis Blut am 30.03.2012 verabschiedet. Es wurde erarbeitet von den Mitgliedern der Untergruppe „Bewertung Blut-assoziierter Krankheitserreger" des Arbeitskreises Blut:

Prof. Dr. Lutz Gürtler, Dr. Ursula Bauerfeind, Dr. Johannes Blümel, Prof. Dr. Reinhard Burger, Prof. Dr. Christian Drosten, Dr. Albrecht Gröner, Dr. Margarethe Heiden, Prof. Dr. Martin Hildebrandt, Prof. Dr. Dr. Bernd Jansen, Dr. Thomas Montag-Lessing, Dr. Ruth Offergeld, Prof. Dr. Georg Pauli, Prof. Dr. Rainer Seitz, Dr. Uwe Schlenkrich, Dr. Volkmar Schottstedt, Dr. Johanna Strobel, Dr. Hannelore Willkommen mit besonderer Unterstützung von Dr. Norbert Bannert (RKI).

\section{Referenzen}

1. Urisman, A., R. J. Molinaro, N. Fischer, S. J. Plummer, G. Casey, E. A. Klein, K. Malathi, C. Magi-Galluzzi, R. R. Tubbs, D. Ganem, R. H. Silverman, and J. L. DeRisi. 2006. Identification of a novel Gammaretrovirus in prostate tumors of patients homozygous for R462Q RNASEL variant. PLoS Pathogens 2:e25.

2. Schlaberg, R., D. J. Choe, K. R. Brown, H. M. Thaker, and I. R. Singh. 2009. XMRV is present in malignant prostatic epithelium and is associated with prostate cancer, especially high-grade tumors. Proc Natl Acad Sci U S A 106:16351-16356.

3. Lombardi, V. C., F. W. Ruscetti, J. Das Gupta, M. A. Pfost, K. S. Hagen, D. L. Peterson, S. K. Ruscetti, R. K. Bagni, C. Petrow-Sadowski, B. Gold, M. Dean, R. H. Silverman, and J. A. Mikovits. 2009. Detection of an infectious retrovirus, XMRV, in blood cells of patients with chronic fatigue syndrome. Science 326:585-589.

4. Lo, S.-C., N. Pripuzova, L. Bingjie, A. L. Komaroff, G.-C. Hung, R. Wang, and H. J. Alter. 2010. Detection of MLV-related virus gene sequences in blood of patients with chronic fatigue syndrome and healthy blood donors. 2010. Proc Natl Acad Sci U S A 107:15874-15879. 
5. Onlamoon, N., J. Das Gupta, P. Sharma, K. Rogers, S. Suppiah, J. Rhea, R. J. Molinaro, C. Gaughan, B. Dong, E. A. Klein, X. Qiu, S. Devare, G. Schochetman, J. Hackett, Jr., R. H. Silverman, and F. Villinger. 2011. Infection, viral dissemination, and antibody responses of rhesus macaques exposed to the human gammaretrovirus XMRV. J Virol 85:4547-4557.

6. Fischer, N., O. Hellwinkel, C. Schulz, F. K. Chun, H. Huland, M. Aepfelbacher, and T. Schlomm. 2008. Prevalence of human gammaretrovirus XMRV in sporadic prostate cancer. J Clin Virol 43:277-283.

7. Fischer, N., C. Schulz, K. Stieler, O. Hohn, C. Lange, C. Drosten, and M. Aepfelbacher. 2010. Xenotropic murine leukemia virus-related gammaretrovirus in respiratory tract. Emerg Infect Dis 16:1000-1002.

8. Erlwein, O., S. Kaye, M. O. McClure, J. Weber, G. Wills, D. Collier, S. Wessely, and A. Cleare. 2010. Failure to detect the novel retrovirus XMRV in chronic fatigue syndrome. PloS One 5:e8519.

9. Groom, H. C., V. C. Boucherit, K. Makinson, E. Randal, S. Baptista, S. Hagan, J. W. Gow, F. M. Mattes, J. Breuer, J. R. Kerr, J. P. Stoye, and K. N. Bishop. 2010. Absence of xenotropic murine leukaemia virus-related virus in UK patients with chronic fatigue syndrome. Retrovirology 7:10.

10. Hohn, O., H. Krause, P. Barbarotto, L. Niederstadt, N. Beimforde, J. Denner, K. Miller, R. Kurth, and N. Bannert. 2009. Lack of evidence for xenotropic murine leukemia virus-related virus (XMRV) in German prostate cancer patients. Retrovirology 6:92

11. Hohn, O., K. Strohschein, A. U. Brandt, S. Seeher, S. Klein, R. Kurth, F. Paul, C. Meisel, C. Scheibenbogen, and N. Bannert. 2010. No evidence for XMRV in German CFS and MS patients with fatigue despite the ability of the virus to infect human blood cells in vitro. PloS One 5:e15632.

12. Switzer, W. M., H. Jia, O. Hohn, H. Zheng, S. Tang, A. Shankar, N. Bannert, G. Simmons, R. M. Hendry, V. R. Falkenberg, W. C. Reeves, and W. Heneine. 2010. Absence of evidence of xenotropic murine leukemia virus-related virus infection in persons with chronic fatigue syndrome and healthy controls in the United States. Retrovirology 7:57.

13. van Kuppeveld, F. J., A. S. Jong, K. H. Lanke, G. W. Verhaegh, W. J. Melchers, C. M. Swanink, G. Bleijenberg, M. G. Netea, J. M. Galama, and J. W. van der Meer. 2010. Prevalence of xenotropic murine leukaemia virus-related virus in patients with chronic fatigue syndrome in the Netherlands: retrospective analysis of samples from an established cohort. BMJ 340:c1018.

14. Knox, K., D. Carrigan, G. Simmons, F. Teque, Y. Zhou, J. Hackett, Jr., X. Qiu, K. C. Luk, G. Schochetman, A. Knox, A. M. Kogelnik, and J. A. Levy. 2011. No evidence of murine-like gammaretroviruses in CFS patients previously identified as XMRV-infected. Science 333:94-97.

15. Shin, C. H., L. Bateman, R. Schlaberg, A. M. Bunker, C. J. Leonard, R. W. Hughen, A. R. Light, K. C. Light, and I. R. Singh. 2011. Absence of XMRV Retrovirus and Other Murine Leukemia Virus-Related Viruses in Patients with Chronic Fatigue Syndrome. J Virol 85:7195-7202.

16. Oakes, B., A. K. Tai, O. Cingoz, M. H. Henefield, S. Levine, J. M. Coffin, and B. T. Huber. 2010. Contamination of human DNA samples with mouse DNA can lead to false detection of XMRV-like sequences. Retrovirology 7:109.

17. Robinson, M. J., O. W. Erlwein, S. Kaye, J. Weber, O. Cingoz, A. Patel, M. M. Walker, W. J. Kim, M. Uiprasertkul, J. M. Coffin, and M. O. McClure. 2010. Mouse DNA contamination in human tissue tested for XMRV. Retrovirology 7:108.
18. Erlwein, O., M. J. Robinson, S. Dustan, J. Weber, S. Kaye, and M. O. McClure. 2011. DNA extraction columns contaminated with murine sequences. PloS One 6:e23484.

19. Sato, E., R. A. Furuta, and T. Miyazawa. 2010. An endogenous murine leukemia viral genome contaminant in a commercial RT-PCR kit is amplified using standard primers for XMRV. Retrovirology 7:110.

20. Garson, J. A., P. Kellam, and G. J. Towers. 2011. Analysis of XMRV integration sites from human prostate cancer tissues suggests PCR contamination rather than genuine human infection. Retrovirology $8: 13$.

21. Hue, S., E. R. Gray, A. Gall, A. Katzourakis, C. P. Tan, C. J. Houldcroft, S. McLaren, D. Pillay, A. Futreal, J. A. Garson, O. G. Pybus, P. Kellam, and G. J. Towers. 2010. Disease-associated XMRV sequences are consistent with laboratory contamination. Retrovirology 7:111.

22. Paprotka, T., K. A. Delviks-Frankenberry, O. Cingoz, A. Martinez, H. J. Kung, C. G. Tepper, W. S. Hu, M. J. Fivash, Jr., J. M. Coffin, and V. K. Pathak. 2011. Recombinant origin of the retrovirus XMRV. Science 333:97-101.

23. Sramkoski, R. M., T. G. Pretlow, 2nd, J. M. Giaconia, T. P. Pretlow, S. Schwartz, M. S. Sy, S. R. Marengo, J. S. Rhim, D. Zhang, and J. W. Jacobberger. 1999. A new human prostate carcinoma cell line, 22Rv1. In Vitro Cell Dev Biol Anim 35:403-409.

24. Alberts, B. 2011. Editorial expression of concern. Science 333:35. 